\title{
Successful Conservative Management of Spontaneous Unilateral Adrenal Hemorrhage in Pregnancy
}

\author{
Vineet V Mishra ${ }^{1}$, Preeti A Goyal ${ }^{2}$, Sumesh Choudhary ${ }^{3}$, Rohina Aggarwal ${ }^{4}$, Priyankur Roy ${ }^{5}$
}

\begin{abstract} spontaneous right adrenal hemorrhage and managed conservatively.

Keywords: Adrenal hemorrhage, Pregnancy, Pregnancy-induced hypertension.
\end{abstract}

Spontaneous adrenal hemorrhage (SAH) in the absence of trauma or adrenal tumor is a rare incidence. Its incidence has been reported from $0.14 \%$ to $1.1 \%$ and it usually involves the right adrenal gland. During pregnancy, unilateral SAH has been reported very rarely. We describe a case who presented to us with pregnancy-induced hypertension (PIH) and pain in the right flank region for 1 day and diagnosed as a case of

Journal of South Asian Federation of Menopause Societies (2019): 10.5005/jp-journals-10032-1169
${ }^{1-5}$ Department of Obstetrics and Gynecology, Institute of Kidney
Diseases and Research Center, Institute of Transplantation Sciences,
Ahmedabad, Gujarat, India

Corresponding Author: Vineet V Mishra, Department of Obstetrics and Gynecology, Institute of Kidney Diseases and Research Center, Institute of Transplantation Sciences, Ahmedabad, Gujarat, India, Phone: +91 9426078333, e-mail: vineet.mishra.ikdrc@gmail.com

How to cite this article: Mishra VV, Goyal PA, Choudhary S, et al. Successful Conservative Management of Spontaneous Unilateral Adrenal Hemorrhage in Pregnancy. J South Asian Feder Menopause Soc 2019;7(1):42-43.

Source of support: Nil

Conflict of interest: None

no complaints of flank pain after 1 month. Her repeat CT scan revealed a decreased size of adrenal hemorrhage.

\section{Discussion}

Adrenal hemorrhage is a relatively uncommon condition with a variable and nonspecific presentation. The main symptoms of the condition are hemorrhagic shock, flank pain, and fever. Signs of acute abdomen (including guarding, rigidity or rebound tenderness) have been reported in $15-20 \%$ because of the retroperitoneal location of adrenals. ${ }^{4}$

Although the pathogenesis of adrenal hemorrhage is unclear but in nontraumatic cases, available evidence has implicated adrenocorticotropic hormone (ACTH), adrenal vein spasm, thrombosis, and limited venous drainage of adrenal as the mechanism behind the condition. ${ }^{4}$ Obstetric causes of bilateral adrenal hemorrhage include toxemia of pregnancy, spontaneous abortion, postpartum hemorrhage, twisted ovarian cyst in pregnancy, and more recently described, antiphospholipid antibody syndrome. ${ }^{5}$ The present case had also pregnancy-induced hypertension. Isolated case reports in association with a long-term nonsteroidal anti-inflammatory drug use in uncomplicated pregnancy and neurofibromatosis have been reported.

Ultrasonography examination of the adrenals (including a doppler study) provides useful information regarding hemorrhage 
into the adrenal gland. Several weeks after the acute event, as the hematoma becomes cystic, the central echogenicity associated with adrenal hemorrhage decreases.

Contrast CT scan of the adrenals is the study of choice in demonstrating the adrenal hemorrhage. In pregnancy, MRI is the investigation of choice. The findings suggestive of hemorrhage into the adrenal are adrenal echogenicity with contrast enhancement, streaky appearance of peri-renal fat, and perinephric hematoma. Several weeks after the acute hemorrhage, the CT scan shows a gradual decrease in size and attenuation.

Spontaneous unilateral adrenal hemorrhage during pregnancy has rarely been described. Our patient was presented with a right flank pain and pregnancy-induced hypertension. MRI confirmed the diagnosis of unilateral adrenal hemorrhage. The patient was managed conservatively. A cesarean was done for fetal indication. On follow-up, after 1 month patient's pain got resolved and CT showed resolving hemorrhage.

Spontaneous adrenal hemorrhage may occur in pregnancy in the absence of a trauma or sepsis and can be managed conservatively if patient is hemodynamically stable. Thus, adrenal hemorrhage should be considered in the differential diagnosis of abdominal or flank pain with retroperitoneal hematoma in pregnancy.

\section{Conclusion}

Spontaneous adrenal hemorrhage during pregnancy is a rare condition. It should be considered in the differential diagnosis of pain abdomen in the flank region in pregnant women. Though it can lead to acute adrenal crisis and hemorrhagic shock, it can be managed conservatively if patients' vitals are stable.

\section{References}

1. Gavrilova-Jordan LP, Edmister WB, et al. Spontaneous adrenal hemorrhage during pregnancy: a review of the literature and a case report of successful conservative management. Obstet Gynecol Surv 2005;60(3):191-195. DOI: 10.1097/01.ogx.0000157357.15401.c3.

2. Merkle W. Spontaneous adrenal gland hemorrhage in adults. Urologe A 1986;25:343-346.

3. Kazarians VB, Kausch I, et al. Spontaneous hemorrhage of the adrenal gland during pregnancy. Aktuelle Urol 2007;38(5):403-405. DOI: 10.1055/s-2007-980138.

4. Kovacs KA, Lam YM, et al. Bilateral massive adrenal hemorrhage. Assessment of putative risk factors by case controlled method. Medicine 2001;80:45-53. DOI: 10.1097/00005792-200101000-00005.

5. Espinosa G, Santos E, et al. Adrenal involvement in antiphospholipid syndrome: clinical and immunological characteristic of 86 patients. Medicine 2003;82:106-118. DOI: 10.1097/00005792-20030300000005 . 